\title{
Pharmacological manipulation of cell death: clinical applications in sight?
}

\author{
Douglas R. Green ${ }^{1}$ and Guido Kroemer ${ }^{2}$ \\ 1Department of Immunology, St. Jude Children's Research Hospital, Memphis, Tennessee, USA. \\ ${ }^{2}$ CNRS, UMR8125, Institut Gustave Roussy, Villejuif, France.
}

\begin{abstract}
This series of Reviews on cell death explores the creation of new therapies for correcting excessive or deficient cell death in human disease. Signal transduction pathways controlling cell death and the molecular core machinery responsible for cellular self-destruction have been elucidated with unprecedented celerity during the last decade, leading to the design of novel strategies for blocking pathological cell loss or for killing unwanted cells. Thus, an increasing number of compounds targeting a diverse range of apoptosis-related molecules are being explored at the preclinical and clinical levels. Beyond the agents that are already FDA approved, a range of molecules targeting apoptosis-regulatory transcription factors, regulators of mitochondrial membrane permeabilization, and inhibitors or activators of cell death-related proteases are under close scrutiny for drug development.
\end{abstract}

\section{Introduction}

Apoptosis was originally defined based on morphological features seen as the cell dies: nuclear condensation, nuclear fragmentation, membrane blebbing, cellular fragmentation into membrane-bound bodies, phagocytosis of the dying cell, and lack of an ensuing inflammatory response (1). The final outcome - apoptosis - is generally the result of the activation of a subset of caspase proteases, in particular caspase-3, -6, and -7 (2). These are the "executioner" caspases, and they mediate their effects by cleavage of specific substrates in the cell. The executioner caspases, and, indeed, all of the core components of the apoptosis machinery, often preexist in healthy cells in inactive forms. Activation of the executioner caspase- 3 and -7 by initiator caspase-8, -9 , and -10 defines the best-understood apoptotic pathways, and we focus on these: the death receptor (extrinsic) pathway, and the mitochondrial (intrinsic) pathway.

In the extrinsic pathway, ligation of death receptors (a subset of the TNF receptor [TNFR] family, including TNFR1, CD95, TNFrelated apoptosis-inducing ligad receptor-1 and -2 [TRAIL-R1 and -R2], and probably DR3/TRAMP) causes recruitment and oligomerization of the adapter molecule FADD within the death-inducing signaling complex (DISC). The oligomerized FADD binds initiator caspase- 8 and -10 , causing their dimerization and activation (3).

Most cell death in vertebrates proceeds via the mitochondrial pathway of apoptosis (Figure 1) (4). Here, the executioner caspases are cleaved and activated by the initiator caspase-9. Like other initiator caspases, caspase- 9 can only be activated by dimerization on the adapter molecule Apaf-1. Apaf-1 preexists in the cytosol as a monomer, and its activation depends on the presence of holocytochrome c. Upon binding of cytochrome $c$ to Apaf-1, dATP gains access to a nucleotide-binding site in Apaf-1, inducing a conformational change in the adapter molecule (5). Apaf-1 then oligomerizes into an "apoptosome" that recruits and activates caspase-9 (6). The release of holo-

Nonstandard abbreviations used: AIF, apoptosis-inducing factor; $\mathrm{BH}, \mathrm{Bcl}-2$ homology (domain); DISC, death-inducing signaling complex; MLK, mixed-lineage kinase; MOMP, mitochondrial outer membrane permeabilization; MPT, mitochondrial permeability transition; PARP, poly(ADP-ribose) polymerase; TNFR, TNF receptor; TRAIL, TNF-related apoptosis-inducing ligand; TRAIL-R, TRAIL receptor.

Conflict of interest: The authors have declared that no conflict of interest exists.

Citation for this article: J. Clin. Invest. 115:2610-2617 (2005).

doi:10.1172/JCI26321. cytochrome, which is normally present only in the mitochondrial intermembrane space, is rate-limiting for the generation of the apoptosome. Hence, mitochondrial outer membrane permeabilization (MOMP) is the critical event responsible for caspase activation in the intrinsic pathway. However, MOMP, which represents the "point of no return" of cell death, can even commit a cell to die when caspases are not activated. This "caspase-independent death" $(7,8)$ can occur due to irreversible loss of mitochondrial function and mitochondrial release of caspase-independent death effectors such as apoptosisinducing factor (AIF) (9), endonuclease G $(10)$, and others $(7,8)$.

In ischemic injury and other clinical conditions, MOMP can occur as a consequence of the mitochondrial permeability transition (MPT). Channels in the inner mitochondrial membrane open to allow movement of solutes and ions, resulting in a loss of inner membrane function and swelling of the matrix. This can lead to matrix remodeling, perhaps freeing proteins of the intermembrane space for release, and can lead to outer membrane rupture. The opening of the MPT involves the adenosine nuclear transporter (ANT) in the inner mitochondrial membrane, although this is not absolutely required for MPT (11), and the voltage-dependent anion channels (VDACs) in the outer membrane. Cyclophilin D, located in the matrix, facilitates MPT, probably through enhancing conformational change of inner membrane proteins. Recent studies in cyclophilin D-deficient animals show that this molecule is important for MPT (12-14) and that these mice are resistant to cell death caused by ischemic injury $(12,13)$. However, MOMP and apoptosis induced by several proapoptotic agents remained intact, indicating that a cyclophilin D-independent mechanism (presumably MPT-independent) can also operate in apoptotic death.

This second major mechanism of MOMP involves the Bcl-2 family of proteins. These proteins share 1 or more Bcl-2 homology $(\mathrm{BH})$ domains and control MOMP, mostly at the mitochondrial outer membrane. The proapoptotic multidomain proteins Bax and Bak (containing BH-1, -2, and -3) probably mediate MOMP, as these can permeabilize vesicles, are composed of mitochondrial lipids $(15,16)$, and are required for MOMP to occur during apoptosis (17). Bax and/or Bak can be activated by other $\mathrm{Bcl}-2$ family proteins, the $\mathrm{BH} 3$-only proteins (which share $\mathrm{BH} 3$ ). Some of the proteins, notably Bid and Bim, can activate Bax (and probably Bak) directly, causing MOMP (16). Antiapop- 


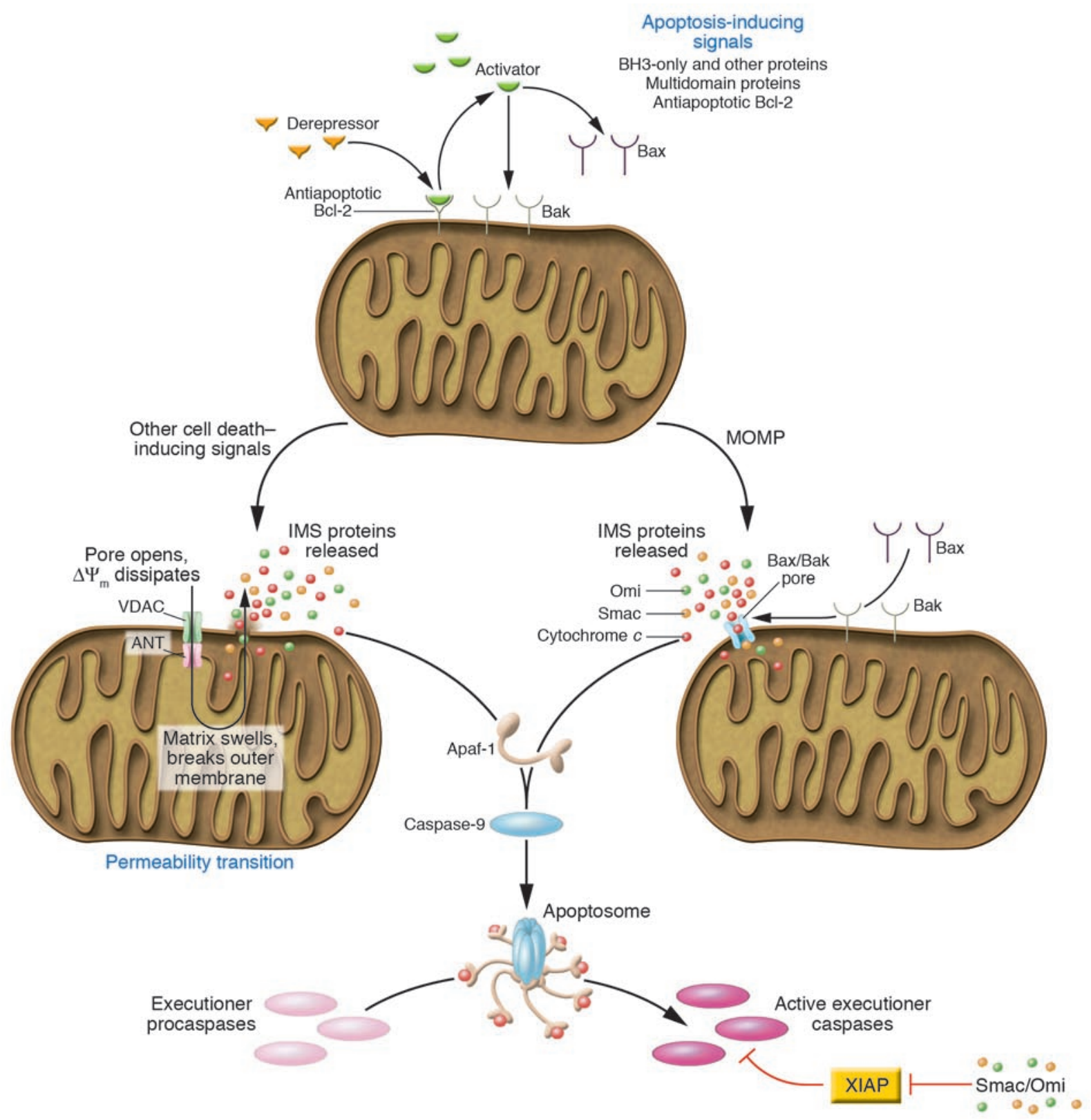

Figure 1

Checkpoints for apoptosis in the mitochondrial pathway. Most mammalian cell death proceeds via the mitochondrial pathway, as illustrated. Stimuli for the induction of apoptosis predominantly act by engaging proapoptotic members of the Bcl-2 family, which work to cause MOMP, and this is countered by the antiapoptotic Bcl-2 family members. Other cell death stimuli can cause MOMP by the induction of a mitochondrial permeability transition. In either case, release of proteins from the intermembrane space triggers the activation of caspases via the formation of an Apaf-1 apoptosome, which recruits and activates caspase- 9 . This, in turn, cleaves and activates the executioner caspases. The activation of caspase- $3,-7$, and -9 is antagonized by XIAP, which in turn can be inhibited by Smac, Omi, and other proteins released upon MOMP. Not shown here are other pathways of caspase activation and apoptosis, including the death receptor pathway, and those resulting in activation of caspase-1 and -2 (see text). ANT, adenosine nuclear transporter; VDAC, voltage-dependent anion channel; IMS, intermembrane space; $\Delta \Psi_{\mathrm{m}}$, mitochondrial transmembrane potential.

totic Bcl-2 family proteins (including Bcl-2, Bcl- $x_{\mathrm{L}}, \mathrm{Mcl}-1$, and others) sequester the $\mathrm{BH} 3$-only proteins and probably the activated multidomain proteins, preventing MOMP. Other BH3only proteins can antagonize the antiapoptotic proteins, thus sensitizing cells for death. These sensitizing or "derepressor" BH3-only proteins include Puma, Noxa, and Bad, among others $(16,18,19)$. Peptides and drugs that mimic the BH3-only proteins therefore have promise as agents that can cause MOMP and apoptosis, or sensitize cells for death (20).

\section{The caveats: apoptosis inhibition without cell death inhibition}

One major problem concerning the development of cell death inhibitors is the definition of cell death and hence the experimental 
Table 1

Therapeutic targets for cell death inhibition at the preclinical stage

\begin{tabular}{|c|c|}
\hline Target & Observations \\
\hline \multicolumn{2}{|l|}{ MOMP } \\
\hline Bcl-2, Bcl- $x_{L}$ & $\begin{array}{l}\text { Intracellular delivery of a plasma membrane-permeable } \mathrm{Bcl}-\mathrm{X}_{\mathrm{L}} \text { protein protects against cerebral infarct in vivo. } \\
\text { A cell-permeable peptide containing the } \mathrm{BH} 4 \text { domain of } \mathrm{Bcl}-\mathrm{X}_{\mathrm{L}} \text { inhibits } \mathrm{x} \text {-ray-induced apoptosis } \\
\text { in the small intestine of mice and partially suppresses anti-CD95-induced fulminant hepatitis. } \\
\text { Transgenic expression of } \mathrm{Bcl}-2 \text { or liposome-mediated delivery of the } \mathrm{Bcl}-2 \text { gene protects against brain ischemia. } \\
\text { Cardiomyocyte-specific transgenic expression of } \mathrm{Bcl}-2 \text { reduces ischemia/reperfusion } \\
\text { injury in the heart and mitigates a mouse model of chronic cardiomyopathy. }\end{array}$ \\
\hline Other proteins & $\begin{array}{l}\text { A variety of agents including cyclosporin A (the prototypic inhibitor of the permeability transition pore) } \\
\text { and inhibitors of mitochondrion-specific ion channels (such as diazoxide, an inhibitor of the MitoKATP channel, } \\
\text { and diltiazem, an inhibitor of } \mathrm{Na}^{+} \mathrm{Ca}^{2+} \text { exchange) inhibit MOMP and exert neuro- and cardioprotective effects } \\
\text { in mouse models. However, the specificity of these agents has not been validated genetically. }\end{array}$ \\
\hline
\end{tabular}

References

Proteases

Caspases Systemic injection of caspase inhibitors reduces the toxicity of neurotoxins (MPTP, malonate, 3-nitrophenol) in vivo. Systemic injection of caspase inhibitors protects against septic shock. Intracochlear infusion of caspase inhibitors reduces cisplatin ototoxicity. Infusion of caspase inhibitors into the inner ear reduces the streptomycin-induced vestibular toxicity.

Calpain A variety of calpain inhibitors protect against ischemic and preservation-reperfusion liver injury, focal cerebral ischemic injury, and traumatic spinal cord injury in rodent models. Transgenic expression of calpastatin reduces neuronal loss induced by MPTP. Knockout of calpastatin sensitizes to kainate-induced neuronal cell loss, while transgenic overexpression of calpastatin reduces cell loss.

Transgenic expression of calpastatin reduces muscle atrophy due to muscle disuse or dystrophin deficiency.

Cathepsins Cathepsin B knockout mice exhibit reduced liver damage in response to TNF- $\alpha$ or cholestasis, and this effect can be mimicked by injection of a chemical cathepsin inhibitor into wild-type mice.

Omi/HtrA2 The Omi/HtrA2-specific inhibitor ucf-101 inhibits the nephrotoxic, proapoptotic effect of cisplatin in mice. Ucf-101 reduces infarct size after myocardial ischemia in mice.

\section{Nonproteolytic death effectors}

AIF Neutralization of AIF can rescue neurons from cell death induced by DNA damage or excitotoxins. Harlequin mice, which bear a hypomorphic AIF mutation, have reduced ischemia/reperfusion damage of the brain.

\section{Death receptors and their ligands}

TNF- $\alpha \quad$ Neutralizing antibodies or soluble receptors are in clinical use for the treatment of rheumatoid arthritis and Crohn disease. CD95L Antibodies specific for CD95L promote regeneration and functional recovery after spinal cord injury in mice. Simultaneous, antibody-mediated neutralization of CD95L and TNF- $\alpha$ reduces infarct volume and mortality in a mouse model of stroke.

Mice lacking CD95L are resistant against neonatal hypoxic-ischemic brain injury and recover locomotor function more rapidly after spinal injury.

DISC Suramin, which inhibits DISC-mediated caspase-8 activation, inhibits apoptotic liver damage induced by CD95 stimulation and endotoxic shock-mediated TNF- $\alpha$ in mice.

\section{Nuclear factors}

p53

An inhibitor of the transactivation function of $p 53$, cyclic pifithrin- $\alpha$ is radioprotective in mice, mimicking the effects of the p53 knockout.

PARP

PARP inhibitors and PARP-1 knockout reduce brain damage induced by stroke or MPTP in mice.

PARP inhibitors and PARP-1 knockout protect against cardiac failure induced by transverse aortic constriction in mice.

\section{Stress kinases}

JNK3

JNK inhibitors reduce infarct size induced by focal ischemia of the rodent brain.

MLK

JNK3 knockout protects the adult mouse brain from glutamate-induced excitotoxicity and cerebral ischemia-hypoxia.

verification of cell death inhibition. It has long been assumed that two decisive events in cellular destruction are apoptotic chromatin condensation and the fragmentation of nuclear DNA detectable with the TUNEL technique. In many instances, the suppression of these nuclear signs of apoptosis has been interpreted as inhibition of cell death. However, inhibition of some signal-transducing events required for apoptotic DNA degradation frequently does not prevent cell death. Thus many studies involving, for instance, caspase inhibitors are flawed when they claim that caspase inhibition prevents cell death without clear demonstration of cellular survival. For example, one of the major DNA-degrading enzymes, caspase-activated DNase (CAD), strictly relies on caspase activation yet is not required for cell 
death, as demonstrated by knockout studies (21). So when CAD is inhibited (which often results in abolition of TUNEL staining), this may indicate that a given compound has achieved caspase inactivation, yet it does not demonstrate that the cell will actually survive. If cell death is evaluated by other techniques - by measurement of MOMP, by assessment of the long-term survival, or by functional assays - then caspase inhibition is often without any effect.

In the context of caspase inhibitors, cells often do not die by apoptosis but succumb to a delayed cell death $(7,8)$ that can have an apoptosis-like morphology (with peripheral chromatin condensation yet without karyorrhexis) (22) or manifest as autophagic cell death (with accumulation of autophagic vacuoles) (23) or necrotic death (with swelling of cytoplasmic organelles) (24). Such "caspaseindependent cell death" is nevertheless under the same regulatory mechanisms upstream of MOMP that control apoptosis (7).

Inhibition of MOMP may have a wider range of cytoprotective actions than inhibition of caspases (4). Accordingly, the antiapoptotic proteins of the $\mathrm{Bcl}-2$ family (which prevent MOMP) have a strong cytoprotective action, even in systems in which caspases play no role in cell death induction (25). However, inhibition of MOMP is not a panacea against cell death. Thus, in conditions in which caspase activation is enforced through the ligation of death receptors or, for instance, by inhibitors of XIAP, cell death can occur without signs of MOMP, and genetic interventions on MOMP-regulatory proteins (such as overexpression of Bcl-2 or knockout of Bax and Bak) may not affect apoptotic cell death. Reportedly, successful MOMP inhibition can also cause a shift from apoptotic to autophagic cell death (26).

\section{Inhibition of cell death at the preclinical and clinical levels}

The death of postmitotic cells in the CNS and myocardium, be it the result of an acute or a chronic degenerative process, is a pathogenic event. Similarly, septic shock, ischemia, and intoxication can cause massive apoptosis in multiple organs. Infections can cause an elevated apoptotic turnover of specific cell types, for instance, lymphocytes in AIDS or gastric mucosa cells in Helicobacter pylori infection. These diseases are therefore candidates for therapeutic cell death suppression.

A cornucopia of putative drugs and targets for cytoprotection are being explored at the preclinical and clinical levels (Tables 1 and 2). MOMP inhibitors have attracted interest, with encouraging results in animal models. Similarly, a variety of protease inhibitors are being designed to prevent cell death. Among this class of agents, caspase inhibitors have been extensively explored. This exploration has been driven by the often-erroneous belief that caspases would generally dictate the "point of no return" of cell death. Although some preclinical results are promising, many of the specific caspase inhibitors (such as zVAD-fmk and zDEVD-fmk) can actually inhibit calpains and cathepsins $(27,28)$. Calpains and cathepsin can also be involved in pathogenic cell death, as indicated by pharmacological studies in rodents and genetic validation in mice (Table 2). Genetic methods to inhibit caspases, for instance by overexpressing the baculovirus protein $\mathrm{p} 35$, have not validated the role of caspases in cell death, for instance in kainate-induced neurotoxicity (29). Moreover, a proof-of-principle experiment illustrating that the conditional or tissue-specific knockout of 1 or several caspase genes can confer true cytoprotection is still elusive. In this sense, it is not surprising that clinical studies of caspase inhibitors assess the treatment of inflammatory diseases such as hepatitis $\mathrm{C}$, in which the inhibition of inflammatory caspases (caspase- 1 and perhaps caspase- 4 and -5) may be the therapeutic goal.

Other strategies of cytoprotection target death-inducing molecules such as TNF- $\alpha$ and CD95L, the proapoptotic transcription factor p53 (which is activated by genotoxic stress as well as by some kinds of metabolic stress), poly(ADP-ribose) polymerase (PARP) (which is activated by DNA damage and hypoxia), or a variety of stress kinases (such as JNK3 or the mixed-lineage kinases [MLKs]) (Tables 1 and 2). Antioxidants may also blunt acute and unwarranted cell death. Some antiapoptotic compounds have been FDA approved (Table 2). This applies in particular to agents targeting TNF- $\alpha$, used to treat rheumatoid arthritis and Crohn disease. Whether such agents act as true cell death blockers or through their antiinflammatory effects, however, is an open question.

\section{Cell damage sensors as targets for cell death induction}

Damage to a variety of cellular organelles can lead to apoptosis. It is conceivable that each organelle possesses sensors that detect specific alterations, locally activate signal transduction pathways, emit signals that ensure interorganellar cross-talk, and ultimately stimulate the common apoptotic pathway, presumably by activating the central executioner (30).

Proteins that belong to the family of PI3K-like kinases sense the DNA damage response. This family includes ATM (ataxia telangiectasia mutated), ATR (ATM- and Rad-3-related), and DNAdependent protein kinase (DNA-PK). In human cells, this directly or indirectly results in the phosphorylation of p53, modulating the DNA-binding activity of $\mathrm{p} 53$ and enhancing its stability. The tumor suppressor protein p53 mediates part of the response of mammalian cells to DNA damage, either by stimulating DNA repair or, beyond a certain threshold of DNA damage, by initiating apoptosis. Transcription factor p53 transactivates a number of proapoptotic proteins from the Bcl-2 family (in particular Bax, Bid, Puma, and Noxa), which induce MOMP and release apoptogenic factors from the mitochondrial intermembrane space. p53 may also induce apoptosis in a transcription-independent manner, by direct physical interactions with members of the Bcl-2 family (31-33). Reactivation of mutated $\mathrm{p} 53$ hence is one of the strategies used to kill tumor cells, in which p53 is frequently mutated or inactivated. Thus a reactivation may be mediated by $\mathrm{p} 53$-expressing adenovirus or by small molecules that reestablish the normal conformation of mutated p53.

While DNA damage often induces p53-dependent apoptosis (which implies that p53-mutated tumor cells do not respond to genotoxic agents), there are a variety of techniques to induce apoptosis by damaging organelles other than the nucleus, a priori in a p53-independent fashion. One possibility is to use agents that cause lysosomal membrane permeabilization, leading to the cytosolic release of cathepsins B and D, which are normally secluded in the lumen of lysosomes $(34,35)$. Cathepsins then trigger an apoptotic response that involves MOMP, followed by caspase activation (36). Alternatively, one can attempt to trigger MOMP by agents directly acting on mitochondria, again bypassing p53 dependency (4). Finally, it is possible to trigger apoptosis via the ER, by inducing the unfolded protein response or by stimulating lethal $\mathrm{Ca}^{2+}$ fluxes (30).

Although there are many ways to stimulate apoptotic responses by compounds inducing organelle-specific damage, thus far no agent specifically acting on cytoplasmic organelles has made it into clinical trials for the treatment of neoplasia. However, ligands of so-called death receptors (such as CD95/Fas, TNFR, and the 2 receptors of 
Table 2

Pharmacological inhibitors of apoptosis that are FDA approved or in clinical development

\begin{tabular}{|c|c|c|c|c|c|}
\hline Drug & Company or institution & Type of compound & Target & Status & Indication \\
\hline \multicolumn{6}{|l|}{ MOMP } \\
\hline $\begin{array}{l}\text { Minocycline } \\
\text { Rasagiline (Agilect) }\end{array}$ & $\begin{array}{c}\text { Danbury Pharmacal } \\
\text { Teva }\end{array}$ & $\begin{array}{l}\text { Small compound } \\
\text { Small compound }\end{array}$ & $\begin{array}{l}\text { Mitochondria } \\
\text { Peripheral } \\
\text { benzodiazepine } \\
\text { receptor? }\end{array}$ & $\begin{array}{c}\text { Phase I } \\
\text { FDA approved }\end{array}$ & $\begin{array}{l}\text { Huntington disease } \\
\text { Parkinson disease }\end{array}$ \\
\hline
\end{tabular}

p53

Amifostine (Ethyol)
Small molecule

Small molecule

Small molecule

Small molecule

Vertex/Aventis

VX-740

Death receptors and their ligands
Adalimumab (HUMIRA) Abbott

Infliximab (Remicade) Centocor/

Schering-Plough

Etanercept (Enbrel)

ISIS 104828

Amgen/Wyeth

Isis

\section{PARP}

INO-1001

Nicotine amide

Inotek
Johns Hopkins University

Antioxidants

Edaravone

Mitsubishi-Tokyo

Idebenone

Kinase inhibitors

CEP-1347
Cephalon/H. Lundbeck
Small molecule

Small molecule

Small molecule

Small molecule

Small molecule p53

FDA approved

Reduction of renal cisplatin toxicity in ovarian or non-small cell lung carcinoma; reduction of radiation effects on the parotid gland

$\begin{array}{cc}\text { Caspases } & \text { Phase II } \\ \text { Caspases } & \text { Phase I } \\ \text { Caspase-1 } & \text { Phase II }\end{array}$

Hepatitis C, acute alcoholic hepatitis,

Acute myocardial infarction Rheumatoid arthritis

$\begin{array}{cc}\text { TNF- } \alpha & \text { FDA approved } \\ \text { TNF- } \alpha & \text { FDA approved } \\ & \\ \text { TNF- } \alpha & \text { FDA approved } \\ \text { TNF- } \alpha & \text { Phase II }\end{array}$

Rheumatoid arthritis, psoriasis, ankylosing spondylitis, Crohn disease Rheumatoid arthritis, Crohn disease

Rheumatoid arthritis, Crohn disease Rheumatoid arthritis, Crohn disease, psoriasis

$\begin{array}{ll}\text { PARP } & \text { Phase I } \\ \text { PARP } & \text { Phase I }\end{array}$

Ischemia/reperfusion damage Ataxia telangiectasia

ROS

Phase III; approved in Japan for treatment of stroke

Reperfusion injury after acute myocardial infarction

Friedreich ataxia Phase I

ROS

MLK inhibitor Phase II/III

Parkinson disease

TRAIL) are being evaluated as potential anticancer agents that could bypass the resistance against conventional chemotherapy (Table 3 ).

\section{Cell death induction: the problem of specificity}

It is easy to trigger apoptosis in most vertebrate cells; the problem is to do this in such a way that only those cells we wish to target die. This is the basis for most cancer therapies but also applies to any situation in which a particular cell population is responsible for disease. For example, selective elimination of a subpopulation of immune cells can form the basis of therapy for inflammatory or autoimmune disease, while specific death of hyperplastic fibroblasts can impact connective tissue diseases. Similarly, we can protect cells from death, but again, specificity is required to ensure that such protection does not promote undesirable cell survival, leading to hyperaccumulation or cancer.

One key to specificity may come with a deeper understanding of why a disease-causing cell does not undergo cell death through normal homeostatic mechanisms. For example, it is well estab- lished that signals to enter the cell cycle simultaneously sensitize cells for apoptosis, such that tissue expansion depends on availability of exogenous survival factors (37). A cell that bypasses the homeostatic apoptotic mechanisms (e.g., through mutation or overexpression of cell death regulators) will, in theory, only have interrupted one physiologically relevant pathway of apoptosis, while leaving other pathways intact and with heightened sensitivity. This is likely the reason why agents that broadly target cells for DNA damage, microtubule breakdown, protease dysfunction, or defects in metabolism can have relatively specific effects on cancer cells, forming the basis of many established approaches to cancer therapy. By understanding and targeting such pathways specifically, we can improve therapies with fewer side effects.

\section{Therapeutic cell death induction at the preclinical and clinical levels}

Most cytotoxic agents used in anticancer chemotherapy induce apoptosis, although very few actually directly target the apoptotic 
Table 3

Pharmacological inducers of apoptosis that are FDA approved or in clinical development

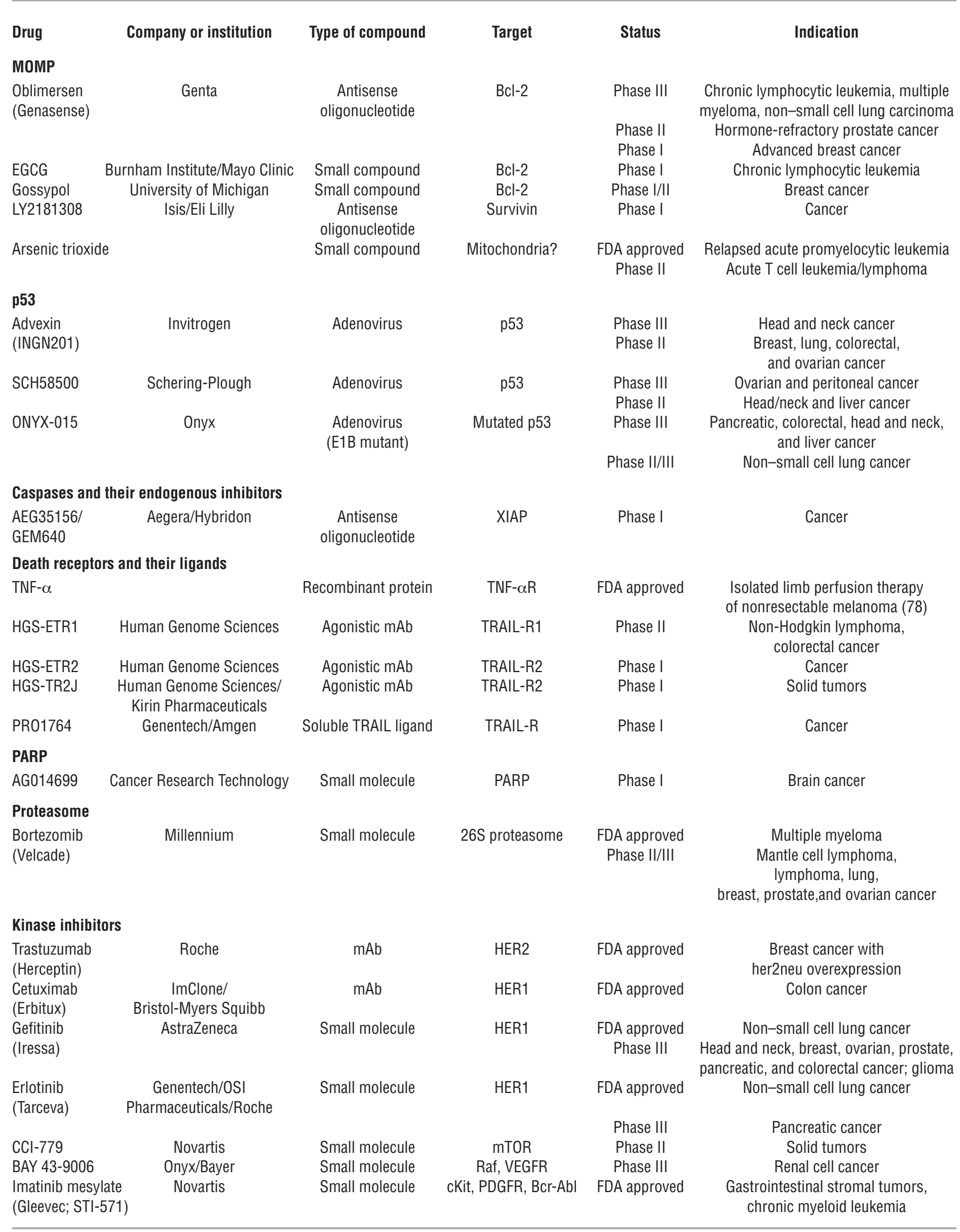


machinery (Table 3). Oblimersen (G3139, Genasense) is an antisense oligonucleotide that targets human $b c l-2$ mRNA. This compound, which has been tested in clinical trials with encouraging results, constitutes the prototype of a new therapeutic concept targeting antiapoptotic molecules. Small molecules targeting Bcl-2 and its relatives are also being developed (20).

p53-expressing adenoviruses as well as an adenovirus designed to replicate in p53-deficient tumor cells (ONYX-015) are also being clinically tested for cancer treatment. TNF- $\alpha$ has been approved for isolated limb perfusion, in which the recombinant protein is injected into an artery of a limb affected by nonresectable melanoma. TRAIL or agonistic antibodies targeting TRAIL-R1 or TRAIL-R2 have entered the early stage of clinical evaluation. A specific PARP inhibitor has also been tested for chemosensitization of brain cancers. More importantly, an inhibitor of the proteasome bortezomib (Velcade) has been approved for chemotherapy of multiple myeloma and is under evaluation as a single agent or in combination chemotherapy for the treatment of hematopoietic and solid cancers (Table 3 ).

However, the true revolution in cancer therapy concerns the therapeutic use of kinase inhibitors targeting protein kinases whose activity is required for cancer cell proliferation and/or survival (Table 3). As an example, one of the prototypic kinase inhibitors, imatinib mesylate (Gleevec), can eradicate BCR/ABL-positive leukemia cells, presumably through the induction of apoptotic (38) or nonapoptotic, caspase-independent (39) cell death. The FDA has approved several other specific inhibitors of survival kinases recently, and dozens are currently under preclinical development.

\section{Conclusions and perspectives: this series}

The current Review Series contains 9 Reviews of areas in cell death research that hold promise for the development of therapeutics.

The Review by Amaravadi and Thompson (40) considers this problem from the perspective of the signaling pathways, in particular the kinases, that control cell survival. This includes not only regulation of apoptosis, per se, but also the signals that control metabolic demand and, in turn, control life and death. Other

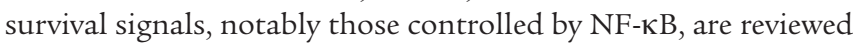
by Luo et al. (41) and considered with respect to cancer therapy. In addition, cellular survival can be preserved by the functions of members of the heat shock protein family, an active area of research that is reviewed by Beere (42). In all of these cases, important targets have been identified, and agents to modulate them are in different stages of development and testing for use in human disease.

As outlined above, the mitochondria sit at the center of a major pathway for cell death controlled by multiple inputs, and this can result in apoptosis or other forms of cell death. Bouchier-Hayes and colleagues (43) consider the mitochondria as a target for pharmacological intervention, especially with respect to control of the Bcl-2 family proteins. Strategies for elaborating specific apoptosis modulators that mimic Bcl-2 family proteins or act on such proteins are discussed by Letai (44). Another organelle that appears to control cell life and death is the ER, which coordinates complex signaling events around calcium storage and the unfolded protein response as well as other forms of ER stress. Xu et al. (45) consider the signal transduction pathways that center on the ER with respect to cell death and its control.

The regulation of caspase activation is, of course, highly relevant to the manipulation of apoptosis, and this subject is reviewed by Lavrik and colleagues (46). Similarly, the IAPs have for some time been thought of predominantly as caspase inhibitors. Recent discoveries have shown that these also play roles distinct from the control of caspases, acting as ubiquitin ligases for other proteins, passenger molecules, and signal transduction proteins. Wright and Duckett (47) consider IAPs as pharmacological targets and how these affect various outcomes in the cell and organism.

Finally, the process of autophagy, distinct from that of apoptosis, accompanies cell death in many cases, and it is possible that this "selfeating" may result in death, or, alternatively, may represent a mechanism for cell survival. Levine and Yuan (48) discuss this process and consider pharmacological approaches to its manipulation.

Many drug targets elucidated in studies of cell death have led to approved pharmacological agents that are currently in use, while many more are in clinical trial. These are promising, of course, but we are only beginning to understand these processes and their potential for clinical benefit. The application of our understanding is now in sight, but the potential extends beyond our vision.

\section{Acknowledgments}

G. Kroemer receives a special grant from the Ligue Nationale contre le cancer as well as grants from the European Community, Fondation de France, Agence Nationale pour la Recherche sur le SIDA, Sidaction, Cancéropôle Ile-de-France, and the French Ministry of Science. D.R. Green is supported by grants from the NIH.

Address correspondence to: Douglas R. Green, Department of Immunology, St. Jude Children's Research Hospital, 332 North Lauderdale Street, Memphis, Tennessee 38105, USA. Phone: (901) 495-3378; Fax: (901) 495-3107; E-mail: douglas.green@stjude.org. Or to: Guido Kroemer, Centre National de la Recherche Scientifique, UMR8125, Institut Gustave Roussy, 39 rue Camille-Desmoulins, F-94805 Villejuif, France. Phone: 331-4211-6046; Fax: 331-4211-6047; E-mail: kroemer@igr.fr.
1. Wyllie, A.H., Kerr, J.F., and Currie, A.R. 1980. Cell death: the significance of apoptosis. Int. Rev. Cytol. 68:251-306.

2. Fuentes-Prior, P., and Salvesen, G.S. 2004. The protein structures that shape caspase activity, specificity, activation and inhibition. Biochem. J. 384:201-232.

3. Debatin, K.M., and Krammer, P.H. 2004. Death receptors in chemotherapy and cancer. Oncogene. 23:2950-2966.

4. Green, D.R., and Kroemer, G. 2004. The pathophysiology of mitochondrial cell death. Science. 305:626-629.

5. Bao, Q., Riedl, S.J., and Shi, Y. 2005. Structure of Apaf- 1 in the auto-inhibited form: a critical role for ADP. Cell Cycle. 4:1001-1003.

6. Acehan, D., et al. 2002. Three-dimensional structure of the apoptosome: implications for assem- bly, procaspase-9 binding, and activation. Mol. Cell. 9:423-432.

7. Chipuk, J.E., and Green, D.R. 2005. Do inducers of apoptosis trigger caspase-independent cell death? Nat. Rev. Mol. Cell Biol. 6:268-275.

8. Kroemer, G., and Martin, S.J. 2005. Caspase-independent cell death. Nat. Med. 11:725-730.

9. Susin, S.A., et al. 1999. Molecular characterization of mitochondrial apoptosis-inducing factor. Nature. 397:441-446.

10. Li, L.Y., Luo, X., and Wang, X. 2001. Endonuclease $\mathrm{G}$ is an apoptotic DNase when released from mitochondria. Nature. 412:95-99.

11. Kokoszka, J.E., et al. 2004. The ADP/ATP translocator is not essential for the mitochondrial permeability transition pore. Nature. 427:461-465.

12. Nakagawa, T., et al. 2005. Cyclophilin D-dependent mitochondrial permeability transition regulates some necrotic but not apoptotic cell death. Nature. 434:652-658

13. Baines, C.P., et al. 2005. Loss of cyclophilin D reveals a critical role for mitochondrial permeability transition in cell death. Nature. 434:658-662.

14. Basso, E., et al. 2005. Properties of the permeability transition pore in mitochondria devoid of Cyclophilin D. J. Biol. Chem. 280:18558-18561.

15. Kuwana, T., et al. 2002. Bid, Bax, and lipids cooperate to form supramolecular openings in the outer mitochondrial membrane. Cell. 111:331-342.

16. Kuwana, T., et al. 2005. BH3 domains of BH3only proteins differentially regulate Bax-mediated mitochondrial membrane permeabilization both directly and indirectly. Mol. Cell. 17:525-535.

17. Wei, M.C., et al. 2001. Proapoptotic BAX and BAK: 
a requisite gateway to mitochondrial dysfunction and death. Science. 292:727-730.

18. Letai, A., et al. 2002. Distinct BH3 domains either sensitize or activate mitochondrial apoptosis, serving as prototype cancer therapeutics. Cancer Cell. 2:183-192.

19. Chen, L., et al. 2005. Differential targeting of prosurvival $\mathrm{Bcl}-2$ proteins by their $\mathrm{BH} 3$-only ligands allows complementary apoptotic function. Mol. Cell. 17:393-403.

20. Oltersdorf, T., et al. 2005. An inhibitor of Bcl-2 family proteins induces regression of solid tumours. Nature. 435:677-681.

21. Zhang, J., Liu, X., Scherer, D.C., van Kaer, L., Wang, X., and Xu, M. 1998. Resistance to DNA fragmentation and chromatin condensation in mice lacking the DNA fragmentation factor 45. Proc. Natl. Acad. Sci. U. S. A. 95:12480-12485.

22. Susin, S.A., et al. 2000. Two distinct pathways leading to nuclear apoptosis. J. Exp. Med. 192:571-579.

23. Yu, L., et al. 2004. Regulation of an ATG7-beclin 1 program of autophagic cell death by caspase- 8 . Science. 304:1500-1502.

24. Hirsch, T., et al. 1997. The apoptosis-necrosis paradox. Apoptogenic proteases activated after mitochondrial permeability transition determine the mode of cell death. Oncogene. 15:1573-1582.

25. Xu, P., Rogers, S.J., and Roossinck, M.J. 2004. Expression of antiapoptotic genes bcl-xL and ced-9 in tomato enhances tolerance to viral-induced necrosis and abiotic stress. Proc. Natl. Acad. Sci. U. S. A. 101:15805-15810.

26. Shimizu, S., et al. 2004. A role of Bcl-2 family of proteins in non-apoptotic programmed cell death dependent on autophagy genes. Nat. Cell Biol. 6:1221-1228.

27. Schotte, P., Declercq, W., Van Huffel, S., Vandenabeele, P., and Beyaert, R. 1999. Non-specific effects of methyl ketone peptide inhibitors of caspases. FEBS Lett. 442:117-121.

28. Knoblach, S.M., et al. 2004. Caspase inhibitor z-DEVD-fmk attenuates calpain and necrotic cell death in vitro and after traumatic brain injury. J. Cereb. Blood Flow Metab. 24:1119-1132.

29. Tomioka, M., et al. 2002. In vivo role of caspases in excitotoxic neuronal death: generation and analysis of transgenic mice expressing baculoviral caspase inhibitor, p35, in postnatal neurons. Brain Res. Mol. Brain Res. 108:18-32.

30. Ferri, K.F., and Kroemer, G.K. 2001. Organelle-specific initiation of cell death pathways. Nat. Cell Biol. 3:E255-E263.

31. Marchenko, N.D., Zaika, A., and Moll, U.M. 2000. Death signal-induced localization of $\mathrm{p} 53$ protein to mitochondria. A potential role in apoptotic signaling. J. Biol. Chem. 275:16202-16212.

32. Chipuk, J.E., et al. 2004. Direct activation of Bax by p53 mediates mitochondrial membrane permeabilization and apoptosis. Science. 303:1010-1014.

33. Leu, J.I., Dumont, P., Hafey, M., Murphy, M.E., and George, D.L. 2004. Mitochondrial p53 activates Bak and causes disruption of a Bak-Mcl1 complex. Nat. Cell Biol. 6:443-450

34. Boya, P., et al. 2003. Lysosomal membrane permeabilization induces cell death in a mitochondriondependent fashion. J. Exp. Med. 197:1323-1334.

35. Erdal, H., et al. 2005. Induction of lysosomal membrane permeabilization by compounds that activate p53-independent apoptosis. Proc. Natl. Acad. Sci.U. S. A. 102:192-197.

36. Boya, P., et al. 2003. Lysosomal membrane permeabilization induces cell death in a mitochondriondependent fashion. J. Exp. Med. 197:1323-1334.

37. Green, D.R., and Evan, G.I. 2002. A matter of life and death. Cancer Cell. 1:19-30.

38. le Coutre, P., et al. 1999. In vivo eradication of human
BCR/ABL-positive leukemia cells with an ABL kinase inhibitor. J. Natl. Cancer Inst. 91:163-168.

39. Okada, M., et al. 2004. A novel mechanism for imatinib mesylate-induced cell death of BCR-ABL-positive human leukemic cells: caspase-independent, necrosis-like programmed cell death mediated by serine protease activity. Blood. 103:2299-2307.

40. Amaravadi, R., and Thompson, C.B. 2005. The survival kinases Akt and Pim as potential pharmacological targets. J. Clin. Invest. 115:2618-2624. doi:10.1172/JCI26273.

41. Luo, J.-L., Kamata, H., and Karin, M. 2005. IKK/NF-КB signaling: balancing life and death - a new approach to cancer therapy. J. Clin. Invest. 115:2625-2632. doi:10.1172/JCI26322.

42. Beere, H.M. 2005. Death versus survival: functional interaction between the apoptotic and stress-inducible heat shock protein pathways. J. Clin. Invest. 115:2633-2639. doi:10.1172/JCI26471.

43. Bouchier-Hayes, L., Lartigue, L., and Newmeyer, D.D. 2005. Mitochondria: pharmacological manipulation of cell death. J. Clin. Invest. 115:2640-2647. doi:10.1172/JCI26274.

44. Letai, A. 2005. Pharmacological manipulation of Bcl-2 family members to control cell death. J. Clin. Invest. 115:2648-2655. doi:10.1172/JCI26250.

45. Xu, C., Bailly-Maitre, B., and Reed, J.C. 2005. Endoplasmic reticulum stress: cell life and death decisions. J. Clin. Invest. 115:2656-2664. doi:10.1172/ JCI26373.

46. Lavrik, I.N., Golks, A., and Krammer, P.H. 2005. Caspases: pharmacological manipulation of cell death. J. Clin. Invest. 115:2665-2672. doi:10.1172/ JCI26252.

47. Wright, C.W., and Duckett, C.S. 2005. Reawakening the cellular death program in neoplasia through the therapeutic blockade of IAP function. J. Clin. Invest. 115:2673-2678. doi:10.1172/JCI26251.

48. Levine, B., and Yuan, J. 2005. Autophagy in cell death: an innocent convict? J. Clin. Invest. 115:2679-2688. doi:10.1172/JCI26390.

49. Cao, G., et al. 2002. In vivo delivery of a Bcl-xL fusion protein containing the TAT protein transduction domain protects against ischemic brain injury and neuronal apoptosis. J. Neurosci. 22:5423-5431.

50. Sugioka, R, et al. 2003. BH4-domain peptide from $\mathrm{Bcl}-\mathrm{xL}$ exerts anti-apoptotic activity in vivo. Oncogene. 22:8432-8440

51. Akhtar, R.S., Ness, J.M., and Roth, K.A. 2004. Bcl-2 family regulation of neuronal development and neurodegeneration. Biochim. Biophys. Acta. 1644:189-203.

52. Tanaka, M., et al. 2004. Cardiomyocyte-specific Bcl-2 overexpression attenuates ischemia-reperfusion injury, immune response during acute rejection, and graft coronary artery disease. Blood. 104:3789-3796.

53. Weisleder, N., Taffet, G.E., and Capetanaki, Y. 2004 $\mathrm{Bcl}-2$ overexpression corrects mitochondrial defects and ameliorates inherited desmin null cardiomyopathy. Proc. Natl. Acad. Sci. U. S. A. 101:769-774.

54. Yang, L., et al. 2004. A novel systemically active caspase inhibitor attenuates the toxicities of MPTP, malonate, and 3NP in vivo. Neurobiol. Dis. 17:250-259.

55. Methot, N., et al. 2004. Differential efficacy of caspase inhibitors on apoptosis markers during sepsis in rats and implication for fractional inhibition requirements for therapeutics. J. Exp. Med. 199:199-207.

56. Wang, J., et al. 2004. Caspase inhibitors, but not c-Jun NH2-terminal kinase inhibitor treatment, prevent cisplatin-induced hearing loss. Cancer Res. 64:9217-9224

57. Matsui, J.I., et al. 2003. Caspase inhibitors promote vestibular hair cell survival and function after aminoglycoside treatment in vivo. J. Neurosci.
23:6111-6122.

58. Liu, X., Van Vleet, T., and Schnellmann, R.G. 2004. The role of calpain in oncotic cell death. Annu. Rev. Pharmacol. Toxicol. 44:349-370.

59. Crocker, S.J., et al. 2003. Inhibition of calpains prevents neuronal and behavioral deficits in an MPTP mouse model of Parkinson's disease. J. Neurosci. 23:4081-4091.

60. Takano, J., et al. 2005. Calpain mediates excitotoxic DNA fragmentation via mitochondrial pathways in adult brains: evidence from calpastatin-mutant mice. J. Biol. Chem. 280:16175-16184.

61. Spencer, M.J., and Mellgren, R.L. 2002. Overexpression of a calpastatin transgene in $\mathrm{mdx}$ muscle reduces dystrophic pathology. Hum. Mol. Genet. 11:2645-2655.

62. Guicciardi, M.A., Leist, M., and Gores, G.J. 2004. Lysosomes in cell death. Oncogene. 23:2881-2890.

63. Cilenti, L., et al. 2005. Omi/HtrA2 protease mediates cisplatin-induced cell death in renal cells. Am. J. Physiol. Renal Physiol. 288:F371-F379.

64. Liu, H.R., et al. 2005. Role of Omi/HtrA2 in apoptotic cell death after myocardial ischemia and reperfusion. Circulation. 111:90-96.

65. Cregan, S.P., et al. 2002. Apoptosis-inducing factor is involved in the regulation of caspase-independent neuronal cell death. J. Cell Biol. 158:507-517.

66. Demjen, D., et al. 2004. Neutralization of CD95 ligand promotes regeneration and functional recovery after spinal cord injury. Nat. Med. 10:389-395.

67. Martin-Villalba, A., et al. 2001. Therapeutic neutralization of CD95-ligand and TNF attenuates brain damage in stroke. Cell Death Differ. 8:679-686.

68. Graham, E.M., et al. 2004. Neonatal mice lacking functional Fas death receptors are resistant to hypoxic-ischemic brain injury. Neurobiol. Dis. 17:89-98.

69. Yoshino, O., et al. 2004. The role of Fas-mediated apoptosis after traumatic spinal cord injury. Spine. 29:1394-1404.

70. Eichhorst, S.T., et al. 2004. Suramin inhibits death receptor-induced apoptosis in vitro and fulminant apoptotic liver damage in mice. Nat. Med. 10:602-609.

71. Komarov, P.G., et al. 1999. A chemical inhibitor of p53 that protects mice from the side effects of cancer therapy. Science. 285:1733-1737.

72. Hong, S.J., Dawson, T.M., and Dawson, V.L. 2004. Nuclear and mitochondrial conversations in cell death: PARP-1 and AIF signaling. Trends Pharmacol. Sci. 25:259-264.

73. Xiao, C.V., et al. 2005. Poly(ADP-ribose) polymerase promotes cardiac remodeling, contractile failure and translocation of apoptosis-inducing factor in a murine experimental model of aortic banding and heart failure. J. Pharmacol. Exp. Ther. 312:891-898.

74. Zheng, J., Devalaraja-Narashimha, K., Singaravelu, K., and Padanilam, B.J. 2005. Poly(ADP-ribose) polymerase- 1 gene ablation protects mice from ischemic renal injury. Am. J. Physiol. Renal Physiol. 288:F387-F398.

75. Hirt, L., et al. 2004. D-JNKI1, a cell-penetrating cJun-N-terminal kinase inhibitor, protects against cell death in severe cerebral ischemia. Stroke. 35:1738-1743.

76. Kuan, C.V., et al. 2003. A critical role of neural-specific JNK3 for ischemic apoptosis. Proc. Natl. Acad. Sci. U.S. A. 100:15184-15189.

77. Wang, L.H., Besirli, C.G., and Johnson, E.M., Jr. 2004. Mixed-lineage kinases: a target for the prevention of neurodegeneration. Annu. Rev. Pharmacol. Toxicol. 44:451-474.

78. Grunhagen, D.J., et al. 2004. One hundred consecutive isolated limb perfusions with TNF-alpha and melphalan in melanoma patients with multiple intransit metastases. Ann. Surg. 240:939-947. 